\title{
Monte Carlo first-order design method for anamorphic cinema zoom lenses
}

\author{
David H. Lippman $\odot$, Doran S. Teverovsky, and Julie L. Bentley \\ University of Rochester, The Institute of Optics, Rochester, New York, United States
}

\begin{abstract}
Anamorphic zoom lenses, used extensively in the motion picture industry, pose a significant design challenge, combining the difficulties of designing a high-performance zoom lens with those of designing an anamorphic lens. As a result, considerable emphasis must be placed on the first-order configuration of the starting point design before interfacing with optical design software. A Monte Carlo search method is introduced for generating first-order designs of anamorphic zoom lenses based on two different configurations. The obtained designs possess valid zoom motions and ray trace successfully while satisfying a set of system specifications. This search method offers a time effective and illustrative way of exploring the global solution space of first-order designs for use as starting points on the way to a thick lens, color-corrected final design. The results of such a Monte Carlo search are presented for two types of anamorphic zoom configurations, and a design example is demonstrated. () The Authors. Published by SPIE under a Creative Commons Attribution 4.0 Unported License. Distribution or reproduction of this work in whole or in part requires full attribution of the original publication, including its DOI. [DOI: 10.1117/1 .OE.60.5.051203]
\end{abstract}

Keywords: optical design; zoom lenses; cinema lenses; anamorphic; Monte Carlo.

Paper 20201093SS received Sep. 15, 2020; accepted for publication Nov. 4, 2020; published online Jan. 5, 2021.

\section{Introduction}

The primary function of an imaging system, at its core, is a way of mapping object space to image space. The properties of this mapping, such as the relative sizes of the object and image, are largely determined by a certain characteristic of the imaging system, either effective focal length (EFL) for infinite conjugate objects or magnification for finite conjugate objects. For the typical case of systems that possess rotational symmetry, the EFL or magnification is constant azimuthally about the optical axis, and as a result, the degree of object-image mapping remains constant azimuthally. A constant azimuthal mapping is not a requirement for imaging, however. One may wonder the benefit of having an image mapping that instead varies azimuthally. One such circumstance was explored by Henri Chrétien when designing a periscope for tanks. ${ }^{1,2}$ With more pertinent information appearing laterally in a scene, Chrétien recognized that tank operators required a wide horizontal field-of-view but with as small of an opening as possible in the tank. The solution was a periscope using cylindrical optics to achieve a "stretched" horizontal field-of-view by means of an azimuthally varying image mapping. Chrétien's periscope is an example of an anamorphic optical system, which is defined as a system that is differently powered in two orthogonal planes of symmetry. ${ }^{3}$ In anamorphic systems, the result of such an orthogonal difference in power and an orthogonal difference in image mapping is that images appear spatially compressed and stretched along these perpendicular axes. To contribute power differently along different axes, anamorphic designs require non-rotationally symmetric optical elements. This is most often achieved with cylindrical optical surfaces, which impart optical power along a single axis, but different prism pairs, ${ }^{4,5}$ toroidal surfaces, ${ }^{6}$ and gradient-index optics ${ }^{7}$ are also used. Anamorphic optics have been used for a variety of applications for a very long time, first dating back to the early nineteenth century. ${ }^{8}$

Starting in the mid-twentieth century with "CinemaScope," anamorphic cinema lenses have been used extensively in shooting motion pictures. ${ }^{9}$ Anamorphic lenses were originally developed in cinema to capture widescreen, high aspect ratio images compressed on standardized, and

*Address all correspondence to David H. Lippman, david.lippman@ rochester.edu 


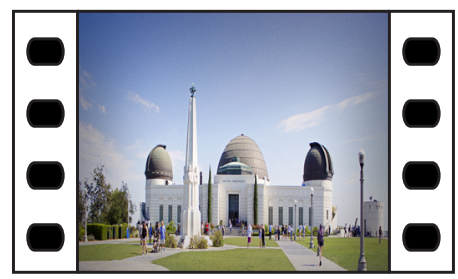

(a)

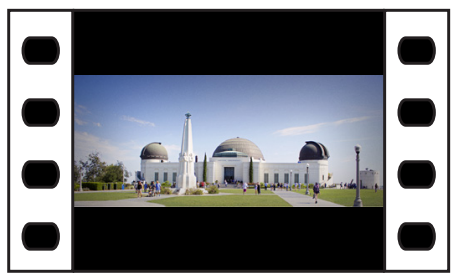

(b)

Fig. 1 Widescreen image captured on a film cell with (a) an anamorphic lens and (b) a rotationally symmetric lens. The anamorphic image is spatially compressed according to the anamorphic ratio and fills the entirety of the film cell. Alternatively, the image from a rotationally symmetric lens must be gated to capture the full widescreen image, but the top and bottom of the film cell go unused.

lower aspect ratio film formats [Fig. 1(a)]. ${ }^{10}$ Doing so achieves higher image resolution and optical throughput than the alternative of shooting the same widescreen format gated, meaning the top and bottom of the film cell go unused [Fig. 1(b)]. Although more customized sensor formats are available today, anamorphic lenses have remained popular in cinema due to their unique image characteristics including elliptical bokeh, ${ }^{11}$ differential depth-of-field, and linear lens flare. A unique aspect of designing cinema lenses is the attention that must be paid to these artistic properties, ${ }^{12}$ the magnitude of which is influenced by the anamorphic ratio, and a critical specification relating the imaging power in the planes of symmetry. Designing anamorphic lenses also poses many new challenges not faced for rotationally symmetric systems, mostly stemming from an expanded set of present aberrations. ${ }^{13,14}$ Most significantly, anamorphic designs must eliminate on-axis astigmatism with both orthogonal planes of symmetry imaging onto a single focal plane. ${ }^{15}$

As discussed, the mapping of object space to image space depends on a first-order characteristic of the imaging system, namely the EFL or magnification depending on the imaging conjugate. A zoom lens is defined as having a continuously variable EFL or magnification, which allows for a continuously variable image mapping without the need for refocusing. This offers a way of continuously varying the field-of-view for a given format and manipulating image properties such as depth of field. This added versatility along with the need for fewer fixed focal length ("prime") lenses explains why zoom lenses are commonplace in the motion picture industry. ${ }^{16}$ The design of zoom lenses has been studied in great detail, particularly regarding the necessary first-order configuration., ${ }^{3,17,18}$ In the simplest case, a zoom system requires two independent moving groups of optical elements, the variator for changing EFL, and the compensator for maintaining a fixed image position. Many different techniques for practically achieving these first-order requirements have been presented including Monte Carlo methods. ${ }^{19-21}$

Due to the prevalence of both anamorphic and zoom lenses in cinema, joint anamorphic zoom lenses are, naturally, also an essential cinematographic tool. Anamorphic zoom lens designs have historically separated the anamorphic elements and the zoom moving groups. ${ }^{22-24}$ This was done for simplicity but at the cost of increased size and weight and more limited aberration correction. The anamorphic module could be placed either in the front or the rear of the rotationally symmetric zoom module, with different optical effects. ${ }^{15}$ The question remained of what possibilities existed for a single combined anamorphic zoom module where the moving optical elements were also the anamorphic elements. The advantage of such a complex design would be a considerable reduction in size and weight of the lens due to the merging of two lens modules into one. This question was recently answered for the first time by Dodoc ${ }^{15}$ who introduced two types of combined anamorphic zoom modules. With such a recent introduction, the potential of these two types of anamorphic zoom modules remains to be fully evaluated.

Individually, the design of high performance anamorphic and zoom lenses is a challenge, so the task of designing a combined anamorphic zoom lens is quite significant. Unlike rotationally symmetric designs, the anamorphic zoom design space is far more insular, making it very difficult to transition between different solution spaces. For these reasons, with the ultimate goal in mind of a final design with satisfactory performance and manufacturing tolerances, considerable 
attention must be paid to the first-order configuration of the starting design. This paper explores the global design space of the two new anamorphic zoom configurations presented by Dodoc. ${ }^{15}$ Using a Monte Carlo search method, the solution spaces of these two design types are fully examined to statistically identify the most promising first-order design configurations. The Monte Carlo search method is presented including how to randomly generate first-order anamorphic zoom solutions tailored to meet system specifications. All generated solutions are then able to be evaluated for successful ray tracing and compared based on various performance metrics. The result yields a close to ideal first-order starting point that will enable the creation of a satisfactory final design for an anamorphic cinema zoom lens.

\section{First-Order Design Configurations}

Both anamorphic and zoom designs present unique difficulties on a first-order level that must be understood individually before attempting combined anamorphic zoom configurations.

With the lost degree of rotational symmetry, anamorphic systems present unique design challenges not found in rotationally symmetric optical systems. Most significantly, the aberration content of anamorphic systems is different than that found in rotationally symmetric systems. For an anamorphic system, the $x-z$ and $y-z$ orthogonal planes of symmetry both possess the Seidel aberrations but in differing amounts. There are, however, eight additional non-rotationally symmetric aberrations originating from skew rays not lying in one of the planes of symmetry, as shown by Yuan and Sasian. ${ }^{13,14}$ On-axis astigmatism is an example of such an aberration seen in anamorphic but not rotationally symmetric systems. The presence of on-axis astigmatism in an anamorphic system can be understood by first considering a single anamorphic element such as a cylindrical lens. For an on-axis point source, a cylindrical lens produces an on-axis line imageoriented orthogonal to the cylindrical axis. To produce a stigmatic image on-axis, a second anamorphic element is required to be oriented orthogonal to the first and must maintain an image position coincident with the image position of the first anamorphic element (see Fig. 2). This elimination of on-axis astigmatism presents a key requirement for valid first-order (VFO) anamorphic configurations, as described in Sec. 3.1. A further consequence of orthogonal anamorphic elements is that there are at most two conjugates for which stigmatic imaging is possible on-axis. ${ }^{13}$ This imposes a limitation on refocusing for different object distances and was the cause of a notorious problem with early anamorphic cinema lenses known as "anamorphic mumps" where close focus positions presented significant on-axis astigmatism. This problem was later solved by Wallin ${ }^{25}$ using an ingenious method of compensating on-axis astigmatism by rotating two cylindrical elements relative to one another.

Zoom systems also present added constraints on first-order designs. By definition, a zoom lens must have a continuously variable EFL (or magnification) while also maintaining a fixed image position. This latter requirement is to ensure an object remains in focus while zooming.

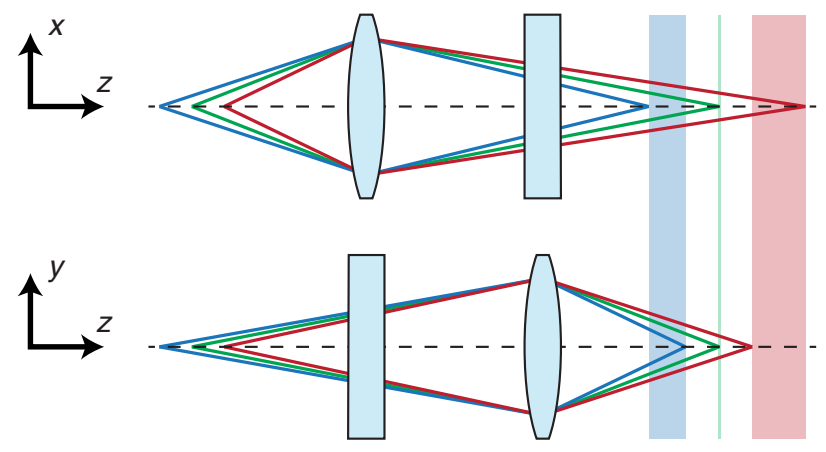

Fig. 2 Presence of on-axis astigmatism for an anamorphic system due to changing conjugates. A single system is shown in the $x-z$ and $y-z$ orthogonal planes of symmetry where cylindrical elements possess power in one axis and no power in the other. Stigmatic imaging occurs only for a single conjugate (green rays). Changing the image conjugate (blue and red rays) introduces on-axis astigmatism. 
In a mechanically compensated zoom, at least two element groups must move independently to meet these requirements, the variator to change EFL and the compensator to maintain a fixed image position. ${ }^{17}$ Additional groups, both fixed and moving, are often employed to provide additional aberration correction, a focusing mechanism, and a fixed total track length (TTL), where TTL is the distance from the first surface vertex to the image. A requirement of all zoom groups is that their movements do not "crash," meaning their positions do not intersect through zoom. This would require groups to change order when zooming, which is not realizable with standard mechanics. Lastly, zoom design configurations are often classified by the order of their groups based on the sign of their power, either positive $(\mathrm{P})$, negative $(\mathrm{N})$, or minimally powered $(\mathrm{X})$. For example, NPX would denote a three-group zoom with a negative, positive, and minimally powered group in that order. The global zoom design space consists of all possible combinations of power signs based on the number of groups.

Focusing now on the design of a combined anamorphic zoom, Dodoc introduced two types of first-order configurations that incorporate the anamorphic components into the zoom moving groups. ${ }^{15}$ Both types are capable of meeting the standard zoom constraints and the constraint of maintaining a constant anamorphic ratio through zoom. Recall, for an infinite conjugate anamorphic system, the anamorphic ratio is defined as the ratio of the system focal lengths in the orthogonal $x-z$ and $y-z$ planes of symmetry:

$$
\mathrm{AR}=\frac{\mathrm{EFL}_{y}}{\mathrm{EFL}_{x}} .
$$

The type I anamorphic zoom configuration [Fig. 3(a)] consists of six zoom groups using two variators and two compensators for a total of four internal moving groups. Using cylindrical elements, one variator-compensator pair operates exclusively in the $x-z$ plane while the other pair operates exclusively in the $y-z$ plane. The stationary front and rear groups are rotationally symmetric and serve in both planes of symmetry as the focus and relay groups, respectively. This means that the difference in system focal length in the $x-z$ and $y-z$ planes according to the anamorphic ratio is entirely attributed to the four anamorphic moving groups (two variatorcompensator pairs). Finally, the aperture stop is located after all moving groups in the relay group to maintain a constant f/number through zoom with a constant iris diameter. The type I configuration is essentially two independent four-group zoom designs in orthogonal planes linked by the anamorphic ratio and two shared stationary groups. As will be seen in Sec. 3.1, two linked four-group zooms are the basis for generating type I first-order solutions.

The type II anamorphic zoom configuration [Fig. 3(b)] consists of five groups with a single spherical variator and two cylindrical compensators oriented orthogonally in $x$ and $y$ for a total of three internal moving groups. As in type I configurations, the first and rear groups are stationary

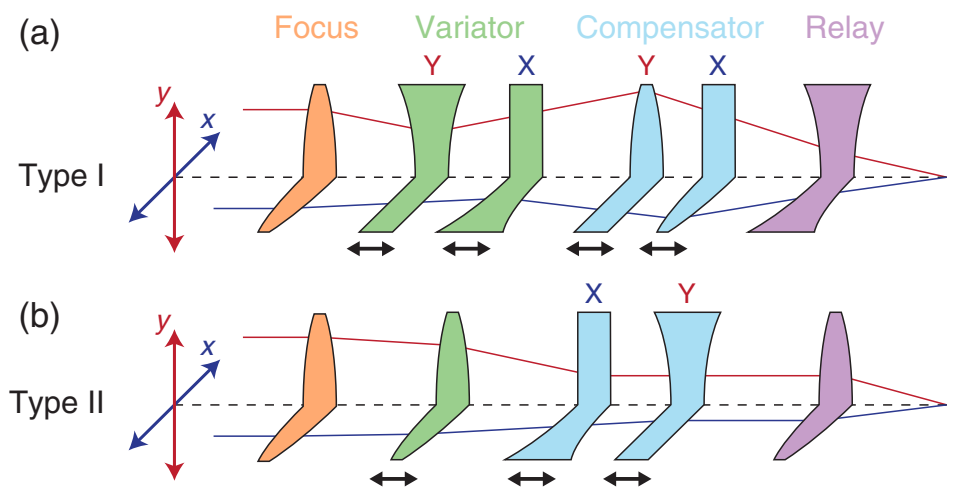

Fig. 3 Type I and type II first-order configurations introduced by Dodoc ${ }^{15}$ for combined anamorphic zoom designs. Both configuration types are depicted with cutouts along the orthogonal planes of symmetry. (a) Type I uses two anamorphic variator-compensators pairs operating in orthogonal planes. (b) Type II uses a spherical variator shared by two anamorphic compensators oriented in orthogonal planes. Both types have fixed focus and relay groups in the front and rear, respectively. The aperture stop is located at the relay group. 
Lippman, Teverovsky, and Bentley: Monte Carlo first-order design method for anamorphic...

Table 1 Number of anamorphic zoom solution spaces for type I and type II first-order design configurations. The total number of solution spaces originates from all possible orderings of group power and cylinder orientation.

\begin{tabular}{lccc}
\hline \hline & Power & Cylinder & Total \\
\hline Type I & 64 & 6 & 384 \\
Type II & 16 & 1 & 16 \\
\hline \hline
\end{tabular}

and used as focusing and relay groups, respectively, where the anamorphic ratio is imparted by the two cylindrical compensators. The aperture stop is again located in the relay group. Unlike in type I, there are three additional constraints on the type II configuration due to the shared spherical variator. These constraints are to ensure there is a constant anamorphic ratio through zoom, as shown by Dodoc. ${ }^{15}$ First, in both orthogonal planes of symmetry, the marginal ray bundle must enter the final (relay) group collimated. Second, the ratio of the cylindrical compensator focal lengths must be equal to the system anamorphic ratio. Third, the separation of the cylindrical compensators must equal their difference in focal length. These additional constraints mean a modified four-group zoom must be used for generating Type II first-order solutions, as done in Sec. 3.1.

For each configuration type, the anamorphic zoom design space is composed of all possible combinations of group power and cylinder orientation orderings (see Table 1). For example, in Fig. 3 the type I configuration is in the PNNPPN-YXYX solution space since the order of the group power signs is PNNPPN and the orientation of the four cylindrical moving groups is YXYX. For this example, it can be seen that the design form is PNPN in both $x$ and $y$, so this solution space can further be abbreviated as PNPN-YXYX. Similarly, the type II configuration in Fig. 3 is in the PPNP-XY solution space. These are just two of the many possible combinations of power and cylinder orderings for both configurations.

For type I, the number of power combinations (either $\mathrm{P}$ or $\mathrm{N}$ ) with six groups is $2^{6}=64$ possible combinations. There are also six different orderings of cylindrical power (either $X$ or $Y$ ), considering two must be in $X$ and two in $Y$. This means for type I there are in total 384 possible first-order solution spaces. It is worth noting that for type I configurations the sign of power is not required to be the same between the two variators and between the two compensators.

For type II, since the ratio of the compensator focal lengths needs to be equal to the system anamorphic ratio, the compensators are required to have the same sign power. This means for type II with five groups but four independent power signs there are $2^{4}=16$ power combinations. Due to the additional compensator constraints on type II configurations, there is only 1 valid ordering of cylindrical elements, depending on the sign of the compensators. For positive compensators the cylinder order must be $Y X$ and for negative compensators the order must be $X Y$. This means for type II there are only 16 possible first-order solution spaces compared with 384 for type I.

\section{Monte Carlo Search}

The objective of the Monte Carlo search is to identify which of the many aforementioned firstorder solution spaces (see Table 1) will offer the best starting point to lead to a satisfactory final design. The Monte Carlo search offers an extremely time effective and informative way of globally inspecting all solution spaces at once rather locally inspecting each individually.

The Monte Carlo search is a three-step process (see Fig. 4). First, given a set of boundary conditions, first-order solutions are randomly generated, and VFO solutions are identified. Next, all VFO solutions are checked for successful thin lens ray tracing across the defined zoom positions, aperture, and field-of-view. The VFO solutions that also successfully ray trace are then identified and saved. Last, the ray traceable (RT) solutions are optimized and evaluated for various performance metrics. The result is a collection of many VFO-RT solutions that can be analyzed and ranked to identify the most successful solution spaces to use as starting points. 


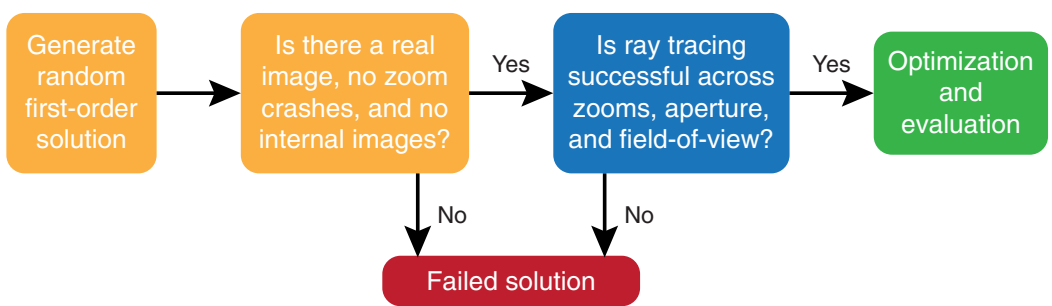

Fig. 4 Flowchart of the Monte Carlo search process. VFO solutions are shown in blue, and RT solutions are in green.

Although applied here to anamorphic cinema zoom lenses, the fundamentals of the presented Monte Carlo search process can be applied to any highly diverse design space as an efficient way of globally searching for starting points. For example, similar processes have been applied in the past to the design of four group zoom lenses ${ }^{19,20}$ and riflescopes. ${ }^{21}$

\subsection{Solution Generation}

The initial step in the Monte Carlo search process is to generate first-order solutions. First, random values are chosen for the group focal lengths, TTL, and back focal length (BFL). The sign of power for the group focal lengths is also chosen at random, except in the case of type II configurations where the compensator focal lengths are constrained by the anamorphic ratio. The magnitude of these randomly selected values is based on boundary conditions provided for allowable group EFL, TTL, and BFL values.

VFO solutions that continue in the Monte Carlo search are those that meet three criteria: (1) they produce a real image, (2) there are no internal images, and (3) there are no zoom group crashes. These requirements are to ensure a properly oriented and accessible image plane and realizable zoom motions. Given the randomly selected group EFLs, TTL, and BFL, a solution's group zoom motions can be calculated using paraxial imaging equations. This is done differently depending on whether a type I or type II configuration is being examined, but both types rely on a four-group zoom motion being applied independently in the $x-z$ and $y-z$ planes. These two four-group zooms are connected by having system focal lengths related by the anamorphic ratio and having the same stationary front and rear groups.

For type I configurations, a standard four-group zoom layout (see Fig. 5) is applied separately in the $x-z$ and $y-z$ planes. The infinite conjugate four-group zoom motion can be derived by repurposing a finite conjugate two-group zoom ${ }^{17}$ as the two internal moving groups over an internal distance $L$. The full four-group zoom is then obtained by adding the stationary front and rear groups to the two-group finite zoom to achieve infinite conjugate imaging, as done by Yee et al. ${ }^{19}$ Given a system EFL zoom range, TTL, BFL, and group focal lengths $f_{1}, f_{2}, f_{3}$, and $f_{4}$, the zoom motions $t_{1}, t_{2}$, and $t_{3}$ of a four-group zoom system are calculated as

$$
\begin{gathered}
\mathrm{VL}=\mathrm{TTL}-\mathrm{BFL}, \\
L=\mathrm{VL}-f_{1}-f_{4} \frac{\mathrm{BFL}}{\mathrm{BFL}-f_{4}}, \\
M=-\mathrm{EFL} \frac{\mathrm{VL}-\mathrm{L}-f_{1}}{\mathrm{BFL} f_{1}}, \\
c=L\left(f_{2}+f_{3}\right)+f_{2} f_{3} \frac{(M-1)^{2}}{M}, \\
t_{1}=V L-L-\frac{f_{4} \mathrm{BFL}}{\mathrm{BFL}-f_{4}}-\frac{t_{2}(M-1)+L}{M-1-M t_{2} / f_{2}},
\end{gathered}
$$




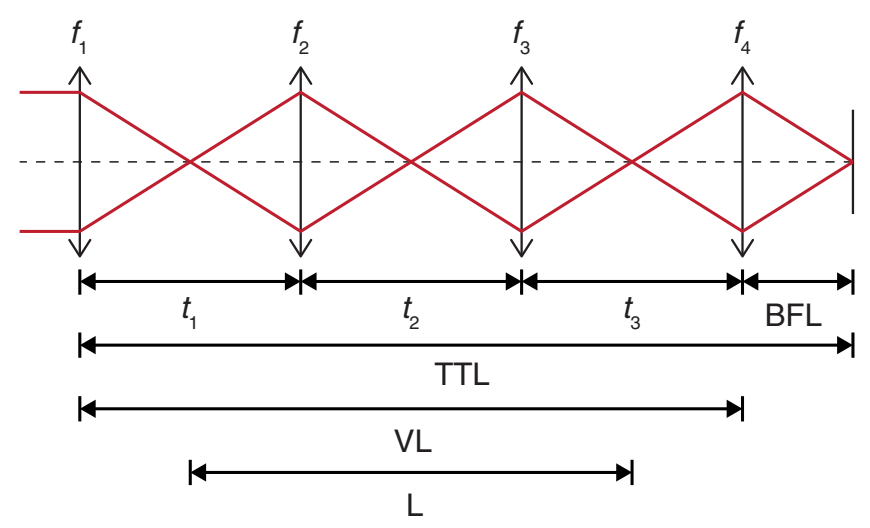

Fig. 5 Standard four-group zoom layout to be applied to type I anamorphic zoom configurations. To obtain a type I configuration, two four-group zooms are obtained in the $x-z$ and $y-z$ planes with different system focal lengths related by the anamorphic ratio and with the same stationary front and rear groups. Real internal images are shown for first-order illustration but are not permitted in the type I configuration.

$$
\begin{gathered}
t_{2}=\frac{L \pm \sqrt{L^{2}-4 c}}{2}, \\
t_{3}=\mathrm{VL}-t_{1}-t_{2} .
\end{gathered}
$$

Focusing on Eq. (7), a valid zoom solution is only possible if the radical is real valued, meaning $L^{2} \geq 4 c$ necessarily for VFO solutions. Also in Eq. (7), the plus-or-minus term means there are potentially two solutions for each configuration for both the positive and negative roots, although it is rarely the case that both solutions have valid zoom motions.

For a type I anamorphic zoom, a first-order design is created using two separate four-group solutions obtained using Eqs. (2)-(8). One four-group system uses the randomly selected values for group EFLs $f_{1}, f_{2, x}, f_{3, x}$, and $f_{4}$ while the system in the orthogonal plane of symmetry uses $f_{1}, f_{2, y}, f_{3, y}$, and $f_{4}$. These two four-group systems are related in several ways. First, the EFL zoom range of these systems is related by the anamorphic ratio, Eq. (1). Second, both four-group systems apply the same stationary front and rear group focal lengths and positions to model the rotationally symmetric focus and relay groups in a type I configuration. Third, applying the same BFL in both planes of symmetry results in a stigmatic image on-axis, as discussed in Sec. 2. With these considerations in mind, these two four-group designs can be joined in the orthogonal planes of symmetry to form a type I first-order solution. The zoom group motions $t_{1, x}, t_{1, y}, t_{2, x}$, $t_{2, y}, t_{3, x}$, and $t_{3, y}$ can now be analyzed to see if there are any group crashes. Examples of a type $\mathrm{I}$ zoom motion for both VFO and crashing solutions can be seen in Fig. 6.

The type II anamorphic zoom configuration relies on a modified four-group zoom (see Fig. 7) applied separately in the $x-z$ and $y-z$ planes. The modifications of the four-group zoom are to meet the requirements on the shared spherical variator discussed in Sec. 2, namely, the marginal ray bundle is collimated into the relay group and the ratio of the compensators in $x$ and $y$ must equal the anamorphic ratio. Given a system EFL zoom range, TTL, BFL, and group focal lengths $f_{1}, f_{2}, f_{3}$, and $f_{4}$, the zoom motions $t_{1}, t_{2}$, and $t_{3}$ for this modified four-group system can similarly be obtained from paraxial imaging equations:

$$
\begin{gathered}
\mathrm{VL}=\mathrm{TTL}-\mathrm{BFL}, \\
M=-\mathrm{EFL} \frac{f_{3}}{f_{1} f_{4}}, \\
t_{1}=f_{1}-f_{2}\left(\frac{1}{M}-1\right), \\
t_{2}=f_{3}+f_{2}(1-M),
\end{gathered}
$$




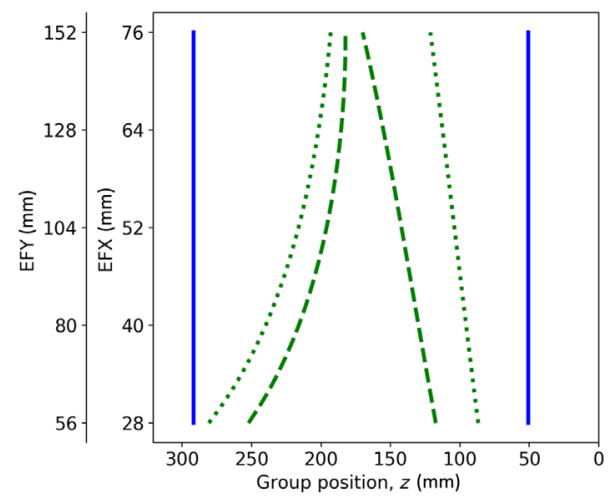

(a)

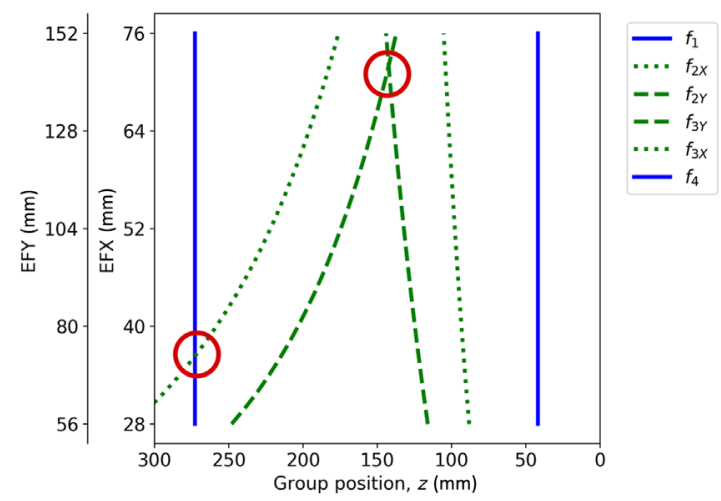

(b)

Fig. 6 Example (a) valid and (b) crashing zoom motions of randomly generated type I first-order solutions in the PNPP-XYYX space. Group position in $z$ (horizontal axis) is shown as a function of system EFL through zoom in $x$ and $y$ (vertical axes) with an anamorphic ratio of 2. The position $z=0 \mathrm{~mm}$ corresponds to the image plane. Stationary groups are shown in blue and moving groups in green. Rotationally symmetric groups are shown with solid lines while anamorphic groups in $X$ are dotted lines and anamorphic groups in $Y$ are dashed. Zoom crashes are circled in red.

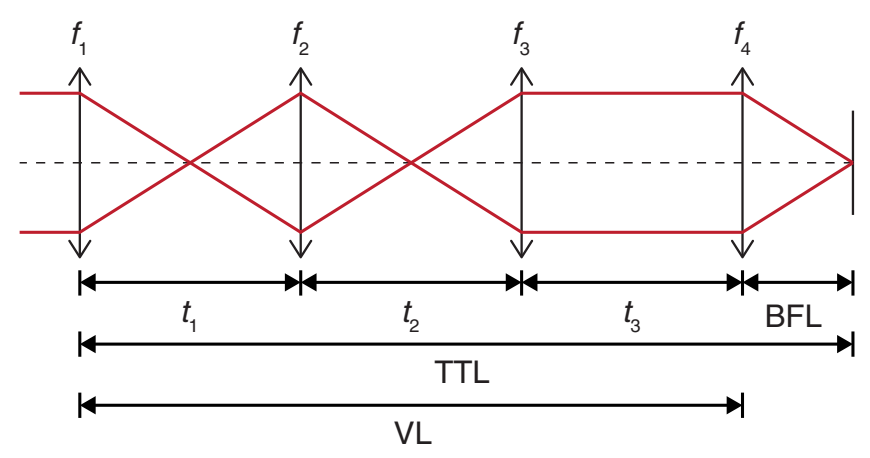

Fig. 7 Modified four-group zoom layout to be applied to type II anamorphic zoom configurations. To achieve a type II configuration, two modified four-group zooms are obtained in the $x-z$ and $y-z$ planes with different system focal lengths related by the anamorphic ratio and with the same stationary front and rear groups. Real internal images are shown for first-order illustration but are not permitted in the type II configuration.

$$
t_{3}=V L-t_{1}-t_{2} .
$$

Unlike with the standard four-group zoom for type I configurations, there is only one possible zoom motion solution for the modified four-group zoom. There is also no constraint due to the realness of a radical.

For a type II anamorphic zoom, a first-order design is created using two separate modified four-group solutions obtained using Eqs. (9)-(13). One modified four-group system uses the randomly selected values for group EFLs $f_{1}, f_{2}, f_{3, x}$, and $f_{4}$ while the system in the orthogonal plane of symmetry uses $f_{1}, f_{2}, f_{3, y}$, and $f_{4}$. As for the type I configuration, the system EFL zoom range of these two four-group systems are related by the anamorphic ratio. Again, both four-group systems apply the same stationary front and rear group focal lengths and positions, including BFL to ensure stigmatic imaging on-axis. Finally, these two modified four-group designs can be joined in orthogonal planes of symmetry to form a type II first-order solution. The zoom group motions $t_{1}, t_{2, x}, t_{2, y}, t_{3, x}$, and $t_{3, y}$ can now be analyzed to see if there are any group crashes. A different aspect of type II configurations is that the compensators in $x$ and $y$ have the same zoom motion with a constant offset equal to their difference in group focal length. 


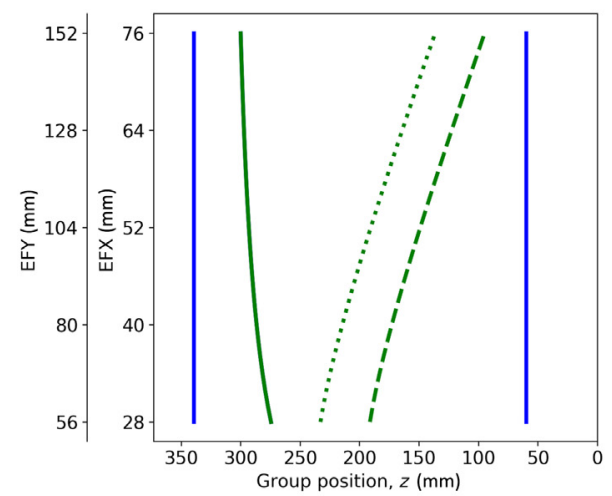

(a)

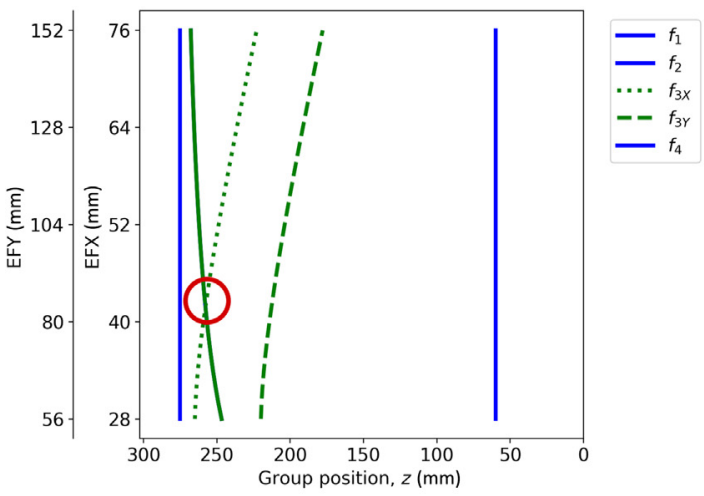

(b)

Fig. 8 Example (a) valid and (b) crashing zoom motions of randomly generated type II first-order solutions in the NPNP-XY space. Group position in $z$ (horizontal axis) is shown as a function of system EFL through zoom in $x$ and $y$ (vertical axes) with an anamorphic ratio of 2 . The position $z=0 \mathrm{~mm}$ corresponds to the image plane. Stationary groups are shown in blue and moving groups in green. Rotationally symmetric groups are shown with solid lines while anamorphic groups in $X$ are dotted lines and anamorphic groups in $Y$ are dashed. Zoom crash is circled in red.

This is a consequence of the added constraints on applying a shared variator. As a result, in assembly the two groups can conveniently be mounted together with the same zoom motion mechanics. As will be seen, however, these added constraints for using a shared variator significantly reduce the design space. Examples of a type II zoom motion for both VFO and crashing solutions can be seen in Fig. 8.

\subsection{Ray Tracing}

After obtaining VFO solutions defined by their group EFLs, TTL, BFL, and zoom motions, the next step in the Monte Carlo search process is to identify successfully RT solutions. A central assumption of the Monte Carlo process is that, for each solution space, the number of found VFO-RT solutions correlates directly with the "size" of the solution space. The size of a solution space is important because it allows for greater flexibility in optimization when attempting to obtain a thick lens, color corrected design. As will be seen, although important, the size of a solution space is not the only factor in determining the best first-order starting point. For example, although a solution space may be very large, this does not necessarily mean that it has better imaging performance than a smaller one.

Ray tracing is performed using the optical design software CODE $\mathrm{V}^{\circledR}$ via an automated process where a VFO zoom solution is modeled using thin lenses. For consistency across designs, each group in the model is composed of three thin lenses in contact with each thin lens contributing one-third of the total group power. By splitting group power, multiple thin lenses in a group improve aberration correction and offer additional variables in optimization. Three thin lenses per group were found to be adequate for analyzing and filtering starting points, although this should be adjusted as necessary for designing beyond first-order, as in Sec. 4.4. In addition, the model is evaluated with real glasses, Schott N-BK7 for positive elements, and Schott N-SF4 for negative elements, although the first-order system is evaluated monochromatically.

For all zoom positions, with the system operating at the design aperture and field-of-view, rays are traced across the entire pupil and field while checking for ray trace failures. Ray trace failures occur for predominantly two reasons. First, severe aberrations may result in rays failing to intersect a surface. These highly aberrated rays may also result in reflection or total internal reflection at a surface. Second, severe pupil aberrations may prevent a ray from successfully being traced between the centers of the pupils and the aperture stop. Pupil aberrations can be mitigated by changing the stop position, but this is not possible under the current requirement of having the stop positioned after all moving groups. If ray trace failure occurs, design optimization and evaluation cannot take place without adjustment, making these VFO solutions very 
unlikely to be useful starting points. As a result, VFO solutions that fail to ray trace are discarded. On the other hand, VFO solutions that successfully ray trace for all zoom positions, pupil coordinates, and fields-of-view are saved and classified as RT. These VFO-RT solutions continue on to the final step in the search process.

\subsection{Optimization and Evaluation}

The identified VFO-RT solutions now reach the third and final step in the Monte Carlo process: optimization and evaluation. Each thin lens design is optimized to minimize geometrical spot size across the field-of-view and across five zoom positions. The only optimization variables are thin lens bending ${ }^{26}$ and image refocusing while the group EFLs, TTL, BFL, and zoom motions are all held constant to remain consistent with the original VFO-RT solution. These limited variables were found to possess adequate degrees of freedom to analyze and filter solutions. When attempting a final, thick lens design (see Sec. 4.4), additional variables can be added for further correction such as the use of different glasses, doublets, or aspheric surfaces.

After optimization, each design is evaluated for geometrical spot size across all fields-of-view and zoom positions, third-order aberration content, and element clear aperture dimensions. These performance metrics provide ample information about both the imaging performance and packaging size of a first-order design and, combined with first-order values such as average group EFL, TTL, and BFL, offer a thorough picture of the VFO-RT solution. By evaluating a large population of solutions, the set of performance metrics can be applied, by extension, to a solution space as a whole.

\section{Results}

\subsection{System Specifications}

The outlined Monte Carlo search process was applied to all possible solution spaces for both type I and type II anamorphic zoom configurations. As discussed by Dodoc, ${ }^{15,18}$ some solution spaces are mathematically incapable of meeting the requirements for solution generation laid out in Sec. 3.1. For example, a solution type with only negatively powered groups is not capable of forming a real image, one of the requirements of a VFO solution. Nevertheless, all solution spaces were considered in the search to demonstrate this fact with the only consequence being lower solution yield per Monte Carlo trial.

The system specifications for all designs in the search can be seen in Table 2 . The system specifications are based on those of standard anamorphic cinema zoom lenses currently available on the market. For example, an anamorphic ratio of 2 is common in the cinema industry and produces desirable elliptical bokeh and differential depth-of-field. ${ }^{11,12}$

Furthermore, the Monte Carlo search boundary values are listed in Table 3. These chosen boundary values are based on several factors. First, the TTL and BFL packaging boundaries were based on the established system specifications. Some allowance was issued for the packaging boundaries due to the later transition from thin to thick lenses. For the group EFL boundary, a large range is required to find diverse solution spaces. Ideally, group EFL values will be large in magnitude to reduce thin lens aberrations, as defined by the G-sums. ${ }^{26}$ Similarly, the minimum group focal length boundary is set to exclude highly aberrated, short focal length groups that are unlikely to lead to RT solutions. An additional consideration is how large of a range should be used for each boundary. Larger boundary ranges entail a more extensive design space will be explored; however, if a range is too large, many Monte Carlo trials will be spent looking at unpromising first-order designs.

\subsection{Type I Configuration}

The type I anamorphic zoom configuration was explored with the Monte Carlo search process using 1 billion trials to examine the 384 possible solution spaces. The total computation time was $\sim 41 \mathrm{~h}$ using a Dell XPS Desktop (8-core i7-9700 @ 3.0 GHz, 32 GB RAM). The results of the 
Lippman, Teverovsky, and Bentley: Monte Carlo first-order design method for anamorphic...

Table 2 System specifications used for the Monte Carlo search. The zoom ratio is the ratio of the longest zoom focal length to the shortest. The projected image aspect ratio of 2.39 is the cinematic anamorphic widescreen format.

\begin{tabular}{lc}
\hline \hline Parameter & Value \\
\hline Anamorphic ratio & 2 \\
EFL, $X(\mathrm{~mm})$ & 28 to 76 \\
EFL, $Y(\mathrm{~mm})$ & 56 to 152 \\
Zoom ratio & 2.7 \\
Image size (mm) & $22.31 \times 18.67$ \\
Image aspect ratio, captured & 1.195 \\
Image aspect ratio, projected & 2.39 \\
Full-field-of-view, horizontal (deg) & 43.4 to 16.7 \\
Full-field-of-view, vertical (deg) & 18.9 to 7.0 \\
F/\# & $F / 4$ \\
Wavelength & Visible \\
TTL (mm) & $\leq 400$ \\
BFL (mm) & $\geq 30$ \\
\hline \hline
\end{tabular}

Table 3 Monte Carlo search boundaries for randomly generated values. The group EFL boundary is unsigned since the power sign for each group EFL is dependent on a trial's randomly assigned solution space.

\begin{tabular}{lc}
\hline \hline Parameter & Value \\
\hline Group EFL, range $(\mathrm{mm})$ & 20 to 500 \\
TTL $(\mathrm{mm})$ & 240 to 360 \\
BFL, range $(\mathrm{mm})$ & 35 to 65 \\
\hline \hline
\end{tabular}

Table 4 Monte Carlo search results of the type I configuration based on whether the obtained solutions have the same design form in $x$ and $y$. For this Monte Carlo search, $10^{9}$ trials were performed.

\begin{tabular}{lcc}
\hline \hline$x, y$ design forms & VFO solutions & RT solutions \\
\hline Same & 10,433 & 491 \\
Different & 7990 & 9 \\
\hline \hline
\end{tabular}

search can be seen in Table 4. It was found that $98.2 \%$ of RT solutions had the same design form in the $x-z$ and $y-z$ planes of symmetry. This is considering that prior to ray tracing, the number of VFO solutions was approximately even with 56.6\% having the same $x$ and $y$ solution types. For example, a PNNPPN-XYXY solution has the same design form, PNPN, in both $x$ and $y$ since the variators and compensators have the same sign power in the orthogonal planes of symmetry. 
Due to the highly skewed findings in favor of solutions with the same design form in the orthogonal planes of symmetry, a revised Monte Carlo search was performed using 1 billion trials and a narrowed scope to exclusively examine solution spaces with the same design form in $x$ and $y$. Based on the overarching assumption that the number of VFO-RT solutions correlates with the size and overall potential of a solution space, the few outlier cases of VFO-RT solutions that had different design forms in $x$ and $y$ were neglected. The reduction in scope from six independent group power signs to four means there is a $4 \times$ reduction in the number of solution spaces, going from 64 to 16 power combinations. The number of cylinder orientation orderings remains unchanged at six, making a total of 96 solutions spaces possible for the type I configuration with the same design form in $x$ and $y$. In addition to increasing solution quality, this narrowing of scope also increased the rate of found RT solutions by $4 \times$.

Considering only solution spaces with the same design form in $x$ and $y$, the revised Monte Carlo search for type I configurations found 41,898 VFO solutions, and of those, 1942 were found to ray trace successfully. This means, of the 1 billion total trials, only $0.002 \%$ resulted in RT solutions, demonstrating just how demanding the design space is for combined anamorphic zooms. For comparison, using a similar method for a standard rotationally symmetric fourgroup zoom, Bruggeman found ${ }^{20} \sim 0.75 \%$ of Monte Carlo trials yielded a RT solution.

The distribution of solution spaces for the type I search presents many relevant findings. The progression of the search process can be seen in Fig. 9, and the breakdown of the quantitative results by solution type can be seen in Fig. 10. Interestingly, there was very little correlation between the power combinations that produced the most VFO solutions and the combinations that produced the most RT solutions. The top three VFO solution spaces are PNPN, NNPN, and NNPP while the top three RT spaces are NPPP, PNPN, and PNPP. Moreover, originally thought to be a weak variable, the order of the cylindrical groups in $x$ and $y$ has a significant impact on what solutions are found. For a certain power ordering, VFO and RT solutions are highly dependent on the cylinder ordering, as opposed to being equally likely for all cylinder orderings. For example, the most common RT solution space NPPP only has RT solutions with cylinder orderings YYXX and YXYX. These findings indicate that the validity of a first-order starting point is equally dependent on the cylinder ordering for a given power ordering as it is on the power ordering itself.

The obtained VFO-RT solutions were optimized as described in Sec. 3.3 and evaluated for a variety of image performance and packaging metrics. The geometrical spot size was calculated and averaged across all field-of-view and zoom positions. The distribution of average spot size by solution space can be seen in Fig. 11. The solution space with the smallest median spot size was NNPP while the space with the smallest absolute spot size was PNPP. The packaging quantities for the designs, namely TTL, BFL, and element clear aperture, were also evaluated on a solution space basis, as shown in Fig. 12. All RT solution spaces had relatively similar TTL and

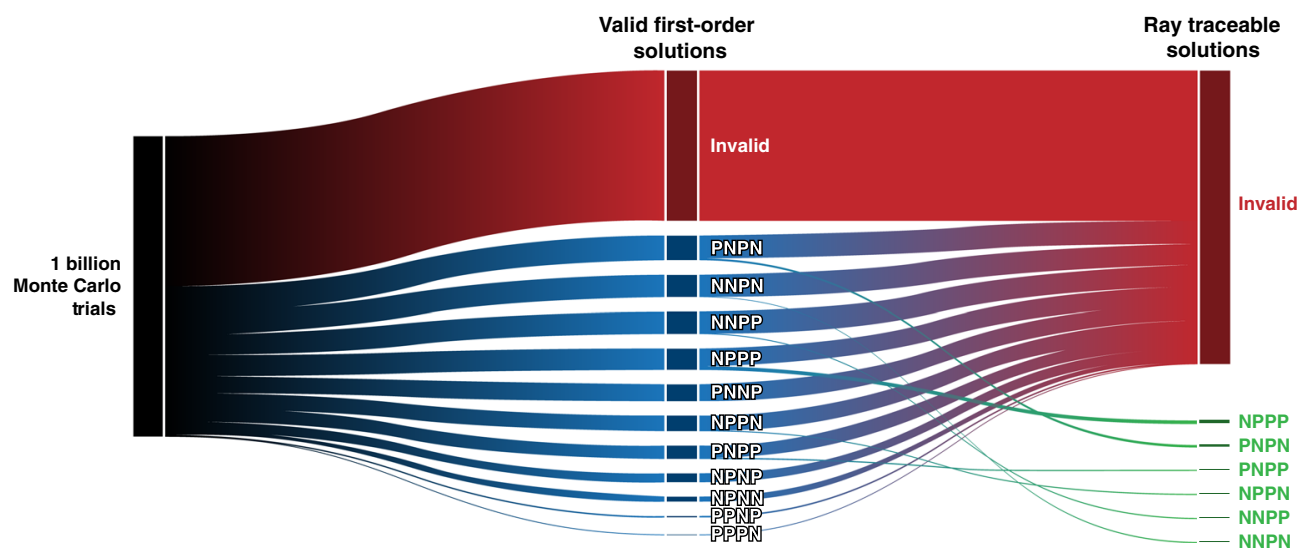

Fig. 9 Monte Carlo search process for the type I anamorphic zoom configuration. Only solutions having the same sign power in $x$ and $y$ were considered for this revised search. VFO solutions are shown in blue, and RT solutions are shown in green. The widths of bands for invalid solutions are not to scale. The quantitative results for found solution types can be found in Fig. 10 . 


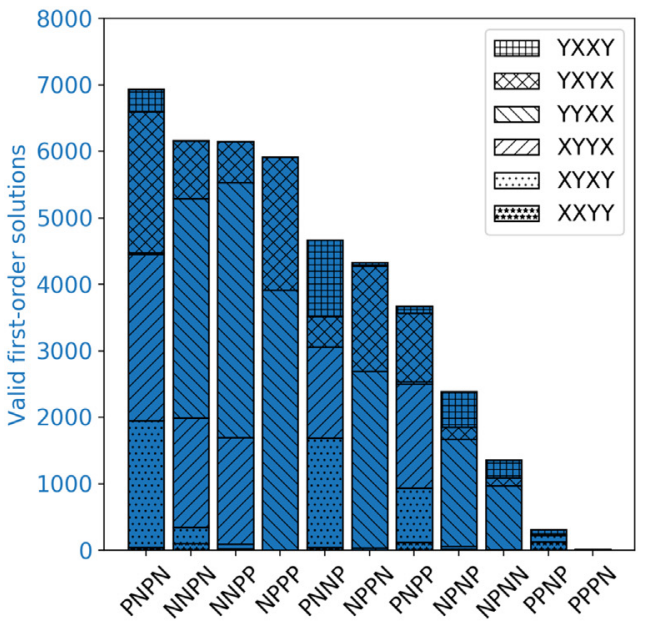

(a)

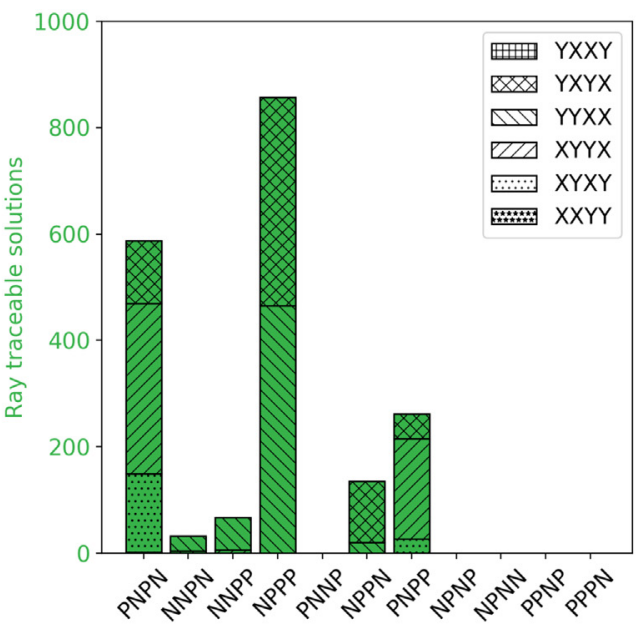

(b)

Fig. 10 Monte Carlo search results for the type I anamorphic zoom configuration. Only solutions having the same sign power in $x$ and $y$ were considered for this revised search. VFO solutions (a) and RT solutions (b) are presented. Solution types are classified by power order (horizontal axis) and orientation ordering (hatch).

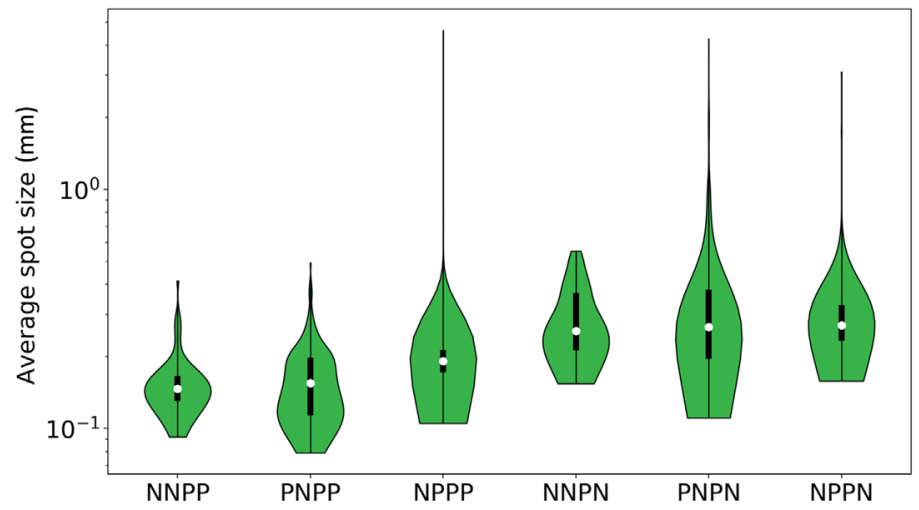

Fig. 11 Average spot size distribution for RT solution spaces of type I configuration. The spot size is averaged over all field-of-view and zoom positions. White points indicate the median while black boxes represents the 25 th to 75 th percentile range. Solution spaces are sorted by median average spot size.
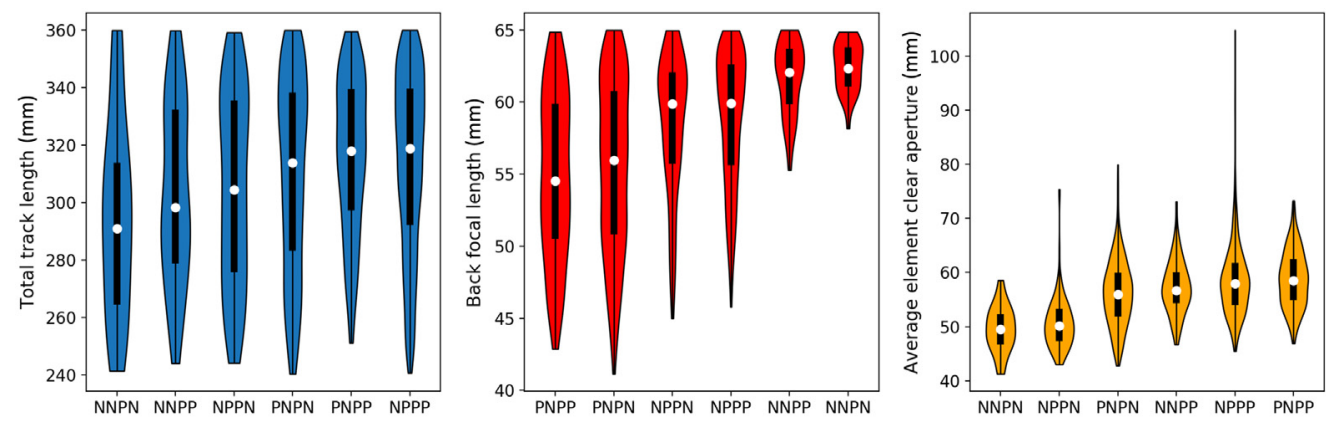

Fig. 12 Packaging distributions for RT solution spaces of type I configuration. Included in packaging is TTL, BFL, and average element clear aperture. TTL is measured from front surface vertex to image. White points indicate the median while black boxes represents the 25th to 75th percentile range. Solution spaces are sorted by median values. 

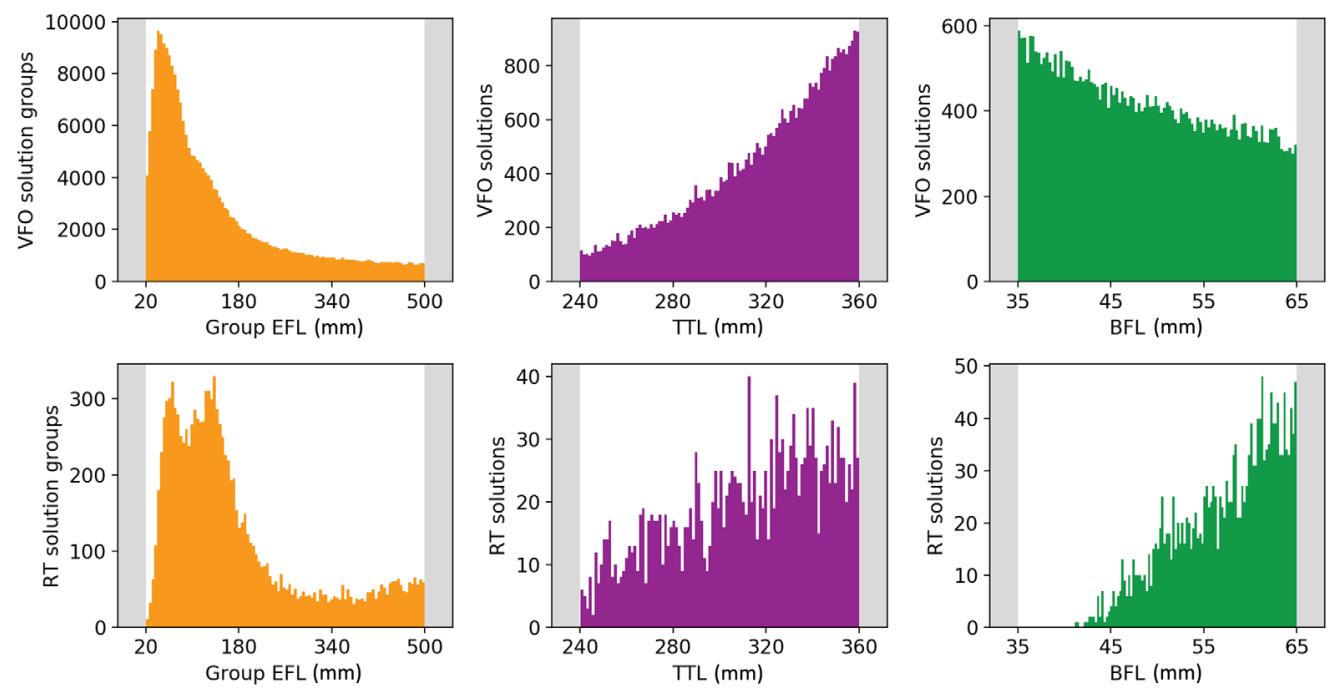

Fig. 13 Distribution of VFO and RT type I solutions according to the search boundary values (see Table 3). The Monte Carlo boundary values for group EFL, TTL, and BFL are shown in gray. The peak of group EFL values shifts larger for RT solutions. For BFL, the trend switches from favoring short values for VFO solutions to long values for RT solutions.

average element clear aperture distributions. On the other hand, BFL distributions varied significantly between spaces.

Another interesting result of the Monte Carlo search is how VFO and RT solutions were distributed relative to the search boundary conditions for group EFL, TTL, and BFL (see Fig. 13). A histogram of group EFL presented a peak for VFO solutions that shifted to longer focal lengths for RT solutions, as you would expect with longer group focal lengths introducing less aberration. For both VFO and RT solutions, there was a trend of more solutions for longer systems, although this trend was less pronounced for RT solutions. Lastly, VFO solutions presented a trend of more solutions for shorter BFL values while RT solutions had the inverse trend of more solutions for longer BFLs.

In summary, the type I configuration offers far more promising results when looking at cases where the design form is the same in both the $x-z$ and $y-z$ planes. Of this set, there were six promising RT solution spaces. Although all six are worth evaluating for a final design, the most promising solution spaces based on size (number of found solutions), imaging performance, and packaging size are PNPP, NPPP, and NNPP. For each of these solution spaces, there are specific cylinder orientation orderings that are required in order to be successful.

\subsection{Type /I Configuration}

The type II anamorphic zoom configuration offers a much more confined design space than the type I configuration. As discussed in Sec. 3.1, due to the shared spherical variator, there are several additional constraints on the group EFLs and zoom motions of the type II configuration. A consequence of these additional constraints is that there are also $24 \times$ fewer solution spaces possible for type II configurations than type I (see Table 1).

The Monte Carlo search was performed for the type II configuration using 100 million trials. From the search, 64,060 valid-first order (VFO) solutions were found; however, solutions in only two spaces were found, 42,537 for NPNP and 21,523 for PNPP. Unfortunately, of these VFO solutions, zero designs ray-traced successfully (see Fig. 14).

The limited number of VFO solution spaces can be attributed to two factors. First, the constraints due to the shared spherical variator limit the number of valid solution spaces. As noted by Dodoc, ${ }^{15}$ of the 16 possible power and cylinder orientation combinations, there are only five solutions spaces that can produce valid zoom motions while satisfying the mathematical framework outlined in Eqs. (9)-(13) for the type II configuration. Second, only two of the five VFO solution spaces were found in the Monte Carlo process due to the search boundary values 


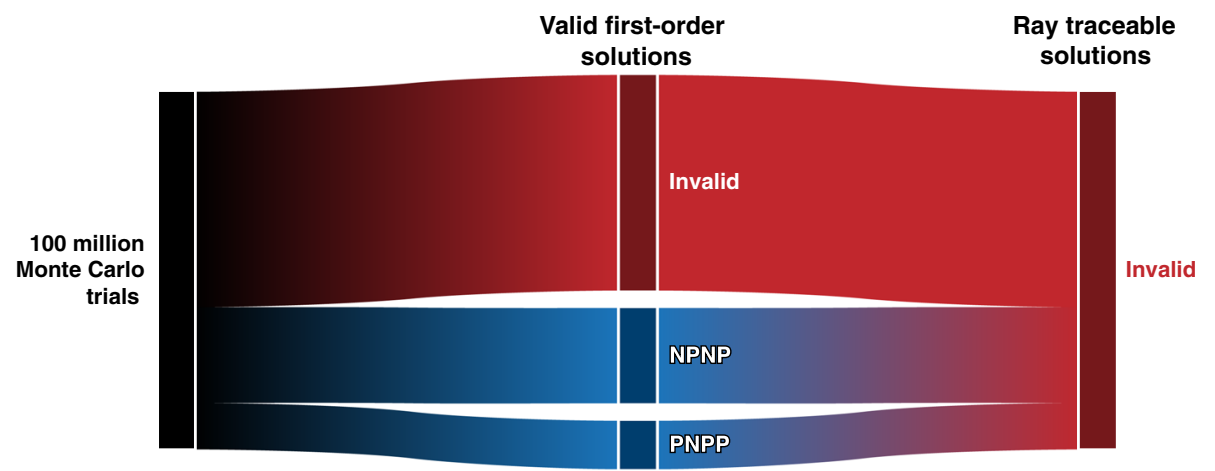

Fig. 14 Monte Carlo search process for the type II anamorphic zoom configuration. Band widths for invalid solutions are not to scale.

(Table 3). For the three valid but not found solution spaces, NNPP designs required BFL $\gtrsim 300 \mathrm{~mm}$, NPPP designs required BFL $\gtrsim 200 \mathrm{~mm}$ and $\left|f_{1}\right| \lesssim 20 \mathrm{~mm}$, and PPNP designs required BFL $\lesssim 20 \mathrm{~mm}$. All of these requirements are beyond the search boundary values as well as the system specifications.

Of the two identified VFO solution spaces, NPNP and PNPP, neither had a single solution that successfully ray traced across all zooms, pupil coordinates, and fields-of-view. This inability to successfully ray trace for the given system specifications is due to the reasons laid out in Sec. 3.2. Finding a VFO solution that satisfies both the field and pupil conjugates with minimal aberration is a challenging problem and, due to the extra constraints on the type II configuration, was not successful for the given system specifications.

Without any RT solution spaces, the type II configuration is not a viable option to consider for a first-order starting point given the system specifications. The type I solution spaces identified in Sec. 4.2 will be used exclusively in obtaining a thick lens, color-corrected final design.
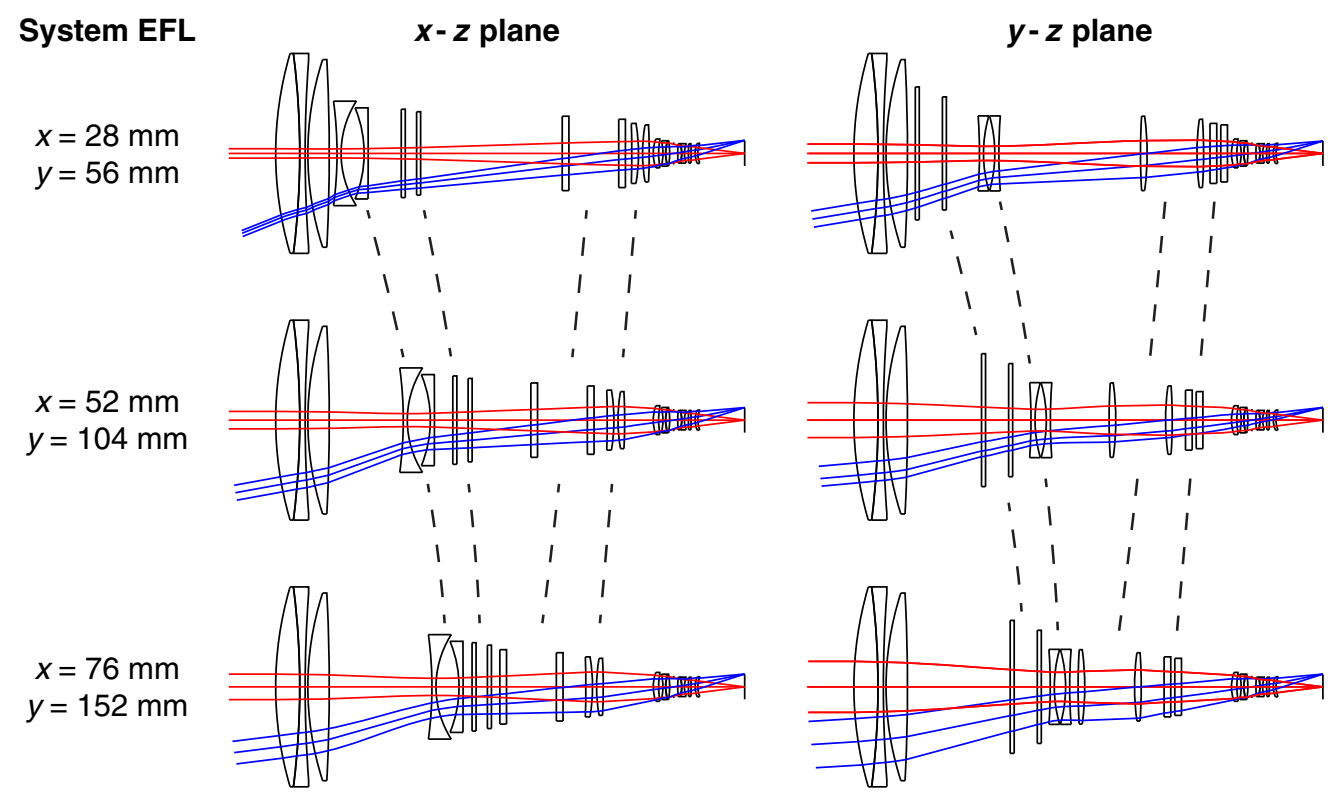

Fig. 15 Final thick lens, polychromatic design using a type I anamorphic zoom configuration in the PNPP-XYYX solution space. The design is shown in the $x-z$ and $y-z$ planes at the short, middle, and longest EFL zoom positions. The design satisfies the system specifications in Table 2 including a TTL of $350 \mathrm{~mm}$ and a BFL of $35 \mathrm{~mm}$. 


\subsection{Final Design}

A final anamorphic design was obtained using a type I configuration in the PNPP-XYYX solution space. Although there are several promising type I solution spaces, the chosen thin lens starting point was a PNPP-XYYX design due to the space's large number of VFO-RT solutions and its good imaging performance (see Figs. 10 and 11). An example PNPP-XYYX first-order design and zoom motion can be seen in Fig. 6(a).

A final design was realized by thickening the thin lenses and performing color correction with different glass types. Starting with three thin lenses per group, elements were removed, split, or compounded into doublets, as necessary. The final design employs 15 elements including three doublets, and each moving group consists of two singlets (see Fig. 15). No aspheric surfaces were used due to their undesirable effect on the appearance of bokeh. ${ }^{27}$ The design satisfies the system specifications in Table 2, including a TTL of $350 \mathrm{~mm}$ and a BFL of $35 \mathrm{~mm}$. The final design was optimized to meet a set of MTF resolution specifications as well. The quality of this design is in large part due to the first-order Monte Carlo search for classifying different solution spaces. This design is one of several obtained and is the first thick lens combined anamorphic zoom presented since the introduction of the first-order configuration by Dodoc. ${ }^{15}$

\section{Conclusions}

The highly restrictive design space for combined anamorphic zoom cinema lenses requires considerable attention to be paid to the first-order design. The global design space for the two configuration types introduced by Dodoc ${ }^{15}$ was explored with a Monte Carlo search process. First-order solutions were randomly generated to meet a set of system specifications, and the accompanying zoom motions were calculated. Of those, the VFO solutions were ray traced. Those that ray traced successfully at all zooms, pupil coordinates, and fields-of-view were optimized and evaluated for a variety of image performance and packaging metrics. This allowed for the classification and ranking of solution spaces and enabled the creation of a final thick lens, color-corrected design. This Monte Carlo process can also be applied to many other highly demanding optical design spaces when searching for an ideal starting point.

The Python code used for the project is available in the GitHub repository, https://github .com/DHLippman/AnaZoom.

\section{Acknowledgments}

The authors thank Scott Cahall and Georg Nadorff at Moondog Optics for the helpful discussion, and the 2020 University of Rochester Advanced Lens Design class for helping to kick off this project.

\section{References}

1. J.-J. Meusy and A. Williams, "Henri Chrétien, Bernard Natan, and the hypergonar," Film Hist. 15(1), 11-31 (2003).

2. A. Arnulf, "Henri Chretien (1879-1956)," Film Hist. 15, 5-10 (2003).

3. W. J. Smith, Modern Optical Engineering, 4th ed., Ch. 13, pp. 323-328, McGraw Hill, New York (2008).

4. T. Kasuya, T. Suzuki, and K. Shimoda, "A prism anamorphic system for Gaussian beam expander," Appl. Phys. 17, 131-136 (1978).

5. S. D. Fantone, "Anamorphic prism: a new type," Appl. Opt. 30, 5008 (1991).

6. S. Yuan and J. Sasian, "Aberrations of anamorphic optical systems III: the primary aberration theory for toroidal anamorphic systems," Appl. Opt. 49, 6802 (2010).

7. J. M. Stagaman and D. T. Moore, "Laser diode to fiber coupling using anamorphic gradientindex lenses," Appl. Opt. 23, 1730 (1984).

8. A. Bouwers and B. Blaisse, "Anamorphic mirror systems," Opt. Acta Int. J. Opt. 2, 36-42 (1955). 
9. E. M. Di Giulio, E. C. Manderfeld, and G. A. Mitchell, "An historical survey of the professional motion-picture camera," J. SMPTE 76, 665-670 (1967).

10. A. Cox, "Anamorphosing optical system," US 2,720,813 (1955).

11. I. A. Neil, “Anamorphic objective lens," US 10,078,201 B2 (2018).

12. L. A. Neil, "A modern series of cinematographic lenses: from concept to product," Proc. SPIE 0892, 165 (1988).

13. S. Yuan and J. Sasian, "Aberrations of anamorphic optical systems I: the first-order foundation and method for deriving the anamorphic primary aberration coefficients," Appl. Opt. 48, 2574 (2009).

14. S. Yuan and J. Sasian, "Aberrations of anamorphic optical systems II primary aberration theory for cylindrical anamorphic systems," Appl. Opt. 48, 2836 (2009).

15. A. Dodoc, "Anamorphic prime and zoom lenses," Proc. SPIE 11106, 1110603 (2019).

16. E. M. DiGiulio, "Developments in motion-picture camera design and technology-a ten year update," SMPTE J. 85, 481-487 (1976).

17. H. Gross, F. Blechinger, and B. Achtner, Handbook of Optical Systems, Volume 4: Survey of Optical Instruments, Wiley-VCH, Weinheim, Germany (2008).

18. A. Dodoc, "Toward the global optimum in zoom lens design," Proc. SPIE 8488, 848802 (2012).

19. A. J. Yee et al., "New tools for finding first-order zoom lens solutions and the analysis of zoom lenses during the design process," Proc. SPIE 9580, 958006 (2015).

20. M. C. Bruggeman and J. L. Bentley, "Determining optimal first-order focal lengths of zoom lenses through Monte Carlo simulations," Proc. SPIE 11106, 1110605 (2019).

21. K. Tinkham et al., "Optical design of a compact high power riflescope with a large zoom ratio," Proc. SPIE (2018).

22. K. Kirchhoff, "Variable focal length anamorphotic cinecamera systems," US 3,751,136 (1973).

23. R. Hirose, “Anamorphotic lens system," US 3,924,933 (1975).

24. T. Suzuki, "Zoom lens with an anamorphic converter," US 5,668,666 (1997).

25. W. Wallin, “Anamorphosing system," US 2,890,622 (1959).

26. R. Kingslake, Lens Design Fundamentals, 1st ed., Academic Press, San Diego, California (1978).

27. V. P. Sivokon and M. D. Thorpe, "Internal structure of bokeh image in camera lenses with aspheric surfaces," Proc. SPIE 8841, 884100 (2013).

David H. Lippman received his BS degree in optical engineering from The Institute of Optics at the University of Rochester in 2018 . He is currently a $\mathrm{PhD}$ student at The Institute of Optics, researching optical design and metrology. He is a member of SPIE.

Doran S. Teverovsky received his BS degree in optics from The Institute of Optics at the University of Rochester in 2020. He is currently a PhD student at The Institute of Optics, researching optical design of AOSLOs. He is a member of SPIE.

Julie L. Bentley received her BS, MS, and PhD degrees in optics from The Institute of Optics at the University of Rochester. She is currently a professor of optics at the University of Rochester, an optical design consultant, and a fellow of SPIE. 